\title{
OPTIMALITY OF RANDOMIZED TRUNK RESERVATION FOR A PROBLEM WITH A SINGLE CONSTRAINT
}

\author{
X. FAN-ORZECHOWSKI*** AND \\ E. A. FEINBERG, ${ }^{* * *}$ State University of New York at Stony Brook
}

\begin{abstract}
We study an optimal admission of arriving customers to a Markovian finite-capacity queue, e.g. an $\mathrm{M} / \mathrm{M} / c / N$ queue, with several customer types. The system managers are paid for serving customers and penalized for rejecting them. The rewards and penalties depend on customer type. The goal is to maximize the average rewards per unit time subject to the constraint on the average penalties per unit time. We provide a solution to this problem based on Lagrangian optimization. For a feasible problem, we show the existence of a randomized trunk reservation optimal policy with the acceptance thresholds for different customer types ordered according to a linear combination of the service rewards and rejection costs. In addition, we prove that any 1-randomized stationary optimal policy has this structure. In particular, we establish the structure of an optimal policy that maximizes the average rewards per unit time subject to the constraint on the blocking probability of either one of the customer types or a group of customer types pooled together.
\end{abstract}

Keywords: Queue; admission control; semi-Markov decision process; trunk reservation; Lagrangian optimization

2000 Mathematics Subject Classification: Primary 60K25

Secondary $60 \mathrm{~K} 15 ; 60 \mathrm{~K} 30$

\section{Introduction}

In this paper we describe the structure of optimal admission policies for finite-capacity queues, including $\mathrm{M} / \mathrm{M} / c / N$ queues, with a fixed number of customer types. At the arrival epoch a customer can be either rejected or accepted. The latter is possible only if the system is not full. Each customer type $i=1,2, \ldots, m$, where $m$ is the number of customer types, is characterized by three parameters: a Poisson arrival rate $\lambda_{i}$, a reward $r_{i}$ that a customer pays for the service, and a penalty $c_{i}$ paid to a rejected customer. The service times do not depend on the customer types. The goal is to maximize the average rewards per unit time subject to the constraint that the average penalty per unit time does not exceed a certain number. Such problems arise, for example, when the goal is to maximize the average rewards per unit time subject to the quality of service constraint.

A randomized trunk reservation policy $\phi$ is defined by $m$ numbers $M_{i}^{\phi}, 0 \leq M_{i}^{\phi} \leq N-1$, $i=1, \ldots, m$, called the 'thresholds'. Of these thresholds, at most one is not an integer and at least one equals $N-1$. For a number $M$ we denote by $\lfloor M\rfloor$ the integer part of $M$. If the system is controlled by the policy $\phi$, a type- $i$ arrival will be admitted with probability 1 if it sees no more

Received 7 October 2004; revision received 21 December 2005.

* Postal address: Department of Applied Mathematics and Statistics, State University of New York at Stony Brook, Stony Brook, NY 11794-3600, USA.

** Email address: xfan@ams.sunysb.edu

*** Email address: eugene.feinberg@ sunysb.edu 
than $\left\lfloor M_{i}^{\phi}\right\rfloor$ customers in the system, will be rejected if the number of customers in the system exceeds $\left\lfloor M_{i}^{\phi}\right\rfloor+1$, and will be accepted with probability $M_{i}^{\phi}-\left\lfloor M_{i}^{\phi}\right\rfloor$ if there are exactly $\left\lfloor M_{i}^{\phi}\right\rfloor+1$ customers in the system at the time of its arrival. In particular, if the number $M_{i}^{\phi}$ is an integer, a type- $i$ arrival will be admitted if and only if it sees no more than $M_{i}^{\phi}$ customers in the system. Thus, having $M_{i}^{\phi}=N-1$ means that a type- $i$ arrival is admitted whenever the system is not full. A randomized trunk reservation policy $\phi$ is said to be consistent with a function $r^{\prime}$, defined on the set $\{1, \ldots, m\}$, if $r_{i}^{\prime}>r_{j}^{\prime}$ implies that $M_{i}^{\phi} \geq M_{j}^{\phi}, i, j=1, \ldots, m$. If all the thresholds are integers, the randomized trunk reservation policy is simply called a trunk reservation policy. We sometimes write $M_{i}$ instead of $M_{i}^{\phi}$ for the thresholds when there is only one policy in context and no confusion will occur.

In this paper we prove that, if the problem is feasible, then there exists a randomized trunk reservation policy that is consistent with the reward function $r_{i}^{\prime}=r_{i}+\bar{u}_{1} c_{i}$, where $\bar{u}_{1} \geq 0$ is a Lagrange multiplier with respect to the first constraint of the linear programming problem to be formulated here. In addition, Theorem 2.3 below shows that any 1-randomized stationary optimal policy is a randomized trunk reservation policy that is consistent with $r^{\prime}$.

In [21] Miller studied a one-criterion problem for an M/M/c/loss queue when $r_{1}>r_{2}>$ $\cdots>r_{m}$. In this case, there exists an optimal nonrandomized trunk reservation policy that is consistent with $r$. In other words, each threshold $M_{i}$ is an integer and $N-1=$ $M_{1} \geq M_{2} \geq \cdots \geq M_{m}$. Feinberg and Reiman [9] studied a constrained problem with $r_{1}>r_{2}>\cdots>r_{m}$ in which the goal is to maximize average rewards per unit time subject to the constraint that the blocking probability for type-1 customers does not exceed a given level. They proved the existence of an optimal randomized trunk reservation policy with $N-1=M_{1} \geq M_{2} \geq \cdots \geq M_{m}$.

Instead of considering $\mathrm{M} / \mathrm{M} / c /$ loss or $\mathrm{M} / \mathrm{M} / c / N$ queues, Feinberg and Reiman [9] made the more general assumption that the service rate $\mu_{n}$, when there are $n$ customers in the system, does not decrease in $n$. This assumption holds for $\mathrm{M} / \mathrm{M} / c / N$ queues. In this paper, we also consider systems that satisfy this assumption.

This research was initially motivated by the following natural question: what is the solution to the problem with $r_{1}>r_{2}>\cdots>r_{m}$ when the goal is to maximize the average rewards per unit time subject to the constraint that the blocking probability for type- $j$ customers does not exceed a given number? This is a particular case of the problem considered in this paper when $c_{j}=\lambda_{j}{ }^{-1}$ and $c_{i}=0, i \neq j$. Therefore,

$$
r_{i}^{\prime}= \begin{cases}r_{j}+\bar{u}_{1} / \lambda_{j} & \text { if } i=j, \\ r_{i} & \text { otherwise }\end{cases}
$$

Since $\bar{u}_{1} \geq 0$, in view of (1.1) we have $r_{j}^{\prime} \geq r_{j}$. Thus, Corollary 2.2 below implies that, when $r_{1} \geq r_{2} \geq \cdots \geq r_{m}$, for a feasible problem there exists an optimal randomized trunk reservation policy with $M_{1} \geq \cdots \geq M_{j-1} \geq M_{j+1} \geq \cdots \geq M_{m}$ and $M_{j} \geq M_{j+1}$. In other words, the threshold for type- $j$ customers can increase. In the particular case with $j=1$, studied by Feinberg and Reiman [9], the orders $r_{1}^{\prime}>r_{2}^{\prime}>\cdots>r_{m}^{\prime}$ and $r_{1}>r_{2}>\cdots>r_{m}$ coincide and, therefore, we have $M_{1}=N-1$ as the highest threshold. If the constraint limits the blocking probability for several customer types pooled together, then the optimal policy also has a simple structure, described in Corollary 2.5. This corollary implies that if $r_{1}>r_{2}>\cdots>r_{m}$ and there is a constraint on the blocking probability for the customers of types $1,2, \ldots, k$ pooled together, with $k \leq m$, then the optimal policy is again a randomized trunk reservation policy consistent with $r$. 
We remark that our main result, Theorem 2.3, is a statement stronger than that of the mere existence of an optimal randomized trunk reservation policy, which is made in Corollary 2.2. We prove that any randomized optimal stationary policy that uses a randomization procedure in at most one state has a randomized trunk reservation form. We recall that, for 1-constrained semi-Markov or continuous-time Markov decision processes describing the problem considered in this paper, when the problem is feasible there exists a randomized stationary optimal policy that uses a randomization procedure in at most one state; see [5] or [6].

In Feinberg and Reiman [9, Sections 6 and 7], several more predictable optimal policies and optimal nonrandomized strategies were constructed. Similar results can be obtained for the more general problem considered in this paper. In fact, these constructions are valid as long as the optimality of randomized trunk reservation policies is established.

In addition to Miller's [21] classical problem formulation and its constrained version studied by Feinberg and Reiman [9], various versions and generalizations of the admission problem have been studied in the literature. Lippman [19] studied a problem with an infinite number of customer classes. Other early references can be found in the surveys by Crabill et al. [4] and Stidham [28]. Nguyen [22] considered a queueing system with two types of arrival: one type is generated by a Poisson process and the other is an overflow process of an $\mathrm{M} / \mathrm{M} / \mathrm{m} / \mathrm{m}$ queue. Carrizosa et al. [3] studied an $\mathrm{M} / \mathrm{G} / c /$ loss queue with different service distributions for different customer types. The control parameter is the probability to accept an arrival, given that the system has available space. This probability depends on the type of the arrived customer and does not depend on the state of the system. Lewis et al. [15], [16] investigated the bias optimality. Lewis [14] studied a dual admission control scheme for an $\mathrm{M} / \mathrm{M} / 1$ queue, with the service times dependent on customer type. Lin and Ross [17], [18] considered optimal admission control policies with a gatekeeper for $\mathrm{M} / \mathrm{M} / 1 /$ loss queues in which the gatekeeper cannot know the busy/idle status of the server. Piunovskiy [24] studied bicriterion control of the arrival intensity for an $\mathrm{M} / \mathrm{M} / 1$ queue. Admission control problems with customers requiring multiple servers were considered by Kelly [12], Key [13], Ross and Yao [27], Papastavrou et al. [23], and Altman et al. [2]. If service times depend on customer type or different types of customer require different numbers of servers, the problem becomes NP-hard and trunk reservation may not be optimal; see [26, p. 137] and [2]. However, a trunk reservation policy is asymptotically optimal under certain conditions; see [11] and [25]. If each customer requests one server and service times do not depend on customer type then trunk reservation policies are optimal [21], [9] and, in addition, the problem is polynomial, because an optimal policy can be found via linear programming; see, e.g. Theorem 2.1 below. The survey of applications of Markov decision problems to communication networks by Altman [1] provides additional references on admission control.

This paper is organized as follows. We formulate the problem and the main results in Section 2. Following Feinberg and Reiman [9], we formulate the problem as a unichain semiMarkov decision problem with one constraint and with finite state and action sets. In Section 2 we also formulate the linear program (LP) that identifies an optimal policy, and explain the meaning of the constant $\bar{u}_{1}$ as an element of the dual solution to this LP.

Previously, Feinberg and Reiman [9, Corollary 3.7] proved that if $r_{1}>r_{2}>\cdots>r_{m}$, then any optimal stationary policy has a trunk reservation form for an unconstrained problem. In Section 3 we study the unconstrained problem when $r_{1} \geq r_{2} \geq \cdots \geq r_{m}$. This case is important because even if we assume that $r_{1}>r_{2}>\cdots>r_{m}$, it is possible that $r_{i}^{\prime}=r_{j}^{\prime}$ for some $i, j=1, \ldots, m$. In Section 4 we establish the link between optimal policies and appropriate LPs. We describe the geometrical structure of the optimal solutions to related LPs 
in Section 5. That is, we show that the optimal LP solution, which corresponds to a randomized optimal policy, is a convex combination of two vectors corresponding to (nonrandomized) stationary policies, and that these three policies differ at most at one point. In addition, the two nonrandomized stationary policies are optimal for the Lagrangian relaxation of the original problem. In Section 6 we prove the main theorem that describes the structure of the optimal policy.

Lagrangian optimization plays an important role in our analysis. We formulate all the required results on Lagrangian optimization in Appendix B. This appendix is important for the following two reasons: (i) for reference purposes in the main body of the paper, and (ii) to present the version of the results needed here, which the authors did not find in the literature on linear or nonlinear programming.

\section{Problem formulation and main results}

We consider a controlled queue that is a generalization of an $\mathrm{M} / \mathrm{M} / c / N$ queue. The queue has space for at most $N$ customers, where $N$ is a given integer. When there are $n$ customers in the queue the departure rate is $\mu_{n}, n=1, \ldots, N$. The numbers $\mu_{n}, n=1, \ldots, N$, satisfy the condition $\mu_{n-1} \leq \mu_{n}$, with $\mu_{0}=0$ and $\mu_{1}>0$. In particular, for an $\mathrm{M} / \mathrm{M} / c / N$ queue, for some $\mu>0$, we have

$$
\mu_{i}= \begin{cases}i \mu & \text { if } i=1, \ldots, c, \\ c \mu & \text { if } i=c+1, \ldots, N .\end{cases}
$$

There are $m=1,2, \ldots$ types of customer, arriving according to $m$ independent Poisson processes with respective intensities $\lambda_{i}, i=1, \ldots, m$. When a customer arrives, its type becomes known. When there are $N$ customers in the system, the system is full and new arrivals are lost. If the system is not full, upon an arrival of a new customer the decision to accept or reject this customer is made. A positive reward $r_{i}$ is collected upon completion of serving an accepted type- $i$ customer. A nonnegative $\operatorname{cost} c_{i}$ is incurred upon the rejection or loss of an arriving type- $i$ customer. The service time of a customer does not depend on the customer type. Unless otherwise specified, we do not assume that $r_{1} \geq r_{2} \geq \cdots \geq r_{m}$.

Our goal is to maximize the average rewards the system collects per unit time, subject to the constraint on the average costs per unit time. In particular, we are interested in the problem of maximizing the average rewards per unit time subject to the blocking probability constraint for a certain type of customer. In a more particular case, when $r_{1}>\cdots>r_{m}$ and the constraint is the blocking probability for type- 1 customers, this problem was studied by Feinberg and Reiman [9].

Following Feinberg and Reiman [9], we model the problem via a semi-Markov decision process. Since the sojourn times between actions are exponentially distributed, this problem is actually an exponential semi-Markov decision process. We refer the reader to [7] for more details. Notice that this problem can also be formulated as a continuous-time Markov decision process. In order to establish the existence of a randomized stationary optimal policy that uses a randomization procedure in at least one state by using continuous-time Markov decision processes, the only extra technical difficulty is to prove that the controlled process has no absorbing states; see [5]. However, for the sake of consistency, since the results of [9] are based on [5] rather than [6], we continue with the approach of [9], using a semi-Markov decision process model.

Let us define the state space $I=\{0,1, \ldots, N-1\} \cup(\{0,1, \ldots, N\} \times\{1, \ldots, m\})$. If the state of the system is $n=0, \ldots, N-1$, a departing customer leaves $n$ customers in the system. 
Being in the state $(n, i)$ means that an arrival of type $i$ sees $n$ customers in the system. Thus, the state space $I$ represents the departure and arrival epochs.

The action set is $A=\{0,1\}$. For $n=0, \ldots, N-1$ and $i=1, \ldots, m$, we set $A(n, i):=$ $A=\{0,1\}$, where the action 0 means that the type- $i$ arrival should be rejected and the action 1 means that it should be accepted. We also set $A(N, i):=\{0\}$. In any state $n=0, \ldots, N-1$, we set $A(n):=\{0\}$. These are departure epochs and the decision-maker does not decide to accept or reject customers in these states. Therefore, we model these action sets $A(n)$ as singletons.

Let $\tau(s, a)$ denote the average time that the system spends in a state $s \in I$ if an action $a \in A(s)$ is chosen in this state. Let $p\left(s, s^{\prime}, a\right)$ be the transition probability from the state $s$ to $s^{\prime}$ if action $a \in A(s)$ is chosen. For notational convenience, we respectively write $\tau(n)$ and $p(n, s)$ instead of $\tau(n, 0)$ and $p(n, s, 0)$ for $n=0, \ldots, N-1$ and $s \in I$. In addition, let $\Lambda=\sum_{i=1}^{m} \lambda_{i}$.

We have $\tau(n)=\left(\mu_{n}+\Lambda\right)^{-1}$, where $n=0, \ldots, N-1$. Also, for $i=1, \ldots, m$,

$$
\tau((n, i), a)= \begin{cases}\tau(n) & \text { if } a=0 \text { and } n=0, \ldots, N, \\ \tau(n+1) & \text { if } a=1 \text { and } n=0, \ldots, N-1 .\end{cases}
$$

For $n=0, \ldots, N-1$ and $i=1, \ldots, m$,

$$
p(n, s)= \begin{cases}\mu_{n} \tau(n) & \text { if } s=n-1, \\ \lambda_{i} \tau(n) & \text { if } s=(n, i), \\ 0 & \text { otherwise, }\end{cases}
$$

and

$$
p((n, i), s, a)= \begin{cases}p(n, s) & \text { if } a=0, \\ p(n+1, s) & \text { if } a=1 .\end{cases}
$$

For simplicity, let the reward be collected when an arrival is accepted. Therefore,

$$
r(s, a):= \begin{cases}r_{i} & \text { if } s=(n, i), n=0, \ldots, N-1, \text { and } a=1 \\ 0 & \text { otherwise }\end{cases}
$$

and

$$
c(s, a):= \begin{cases}c_{i} & \text { if } s=(n, i), n=0, \ldots, N, \text { and } a=0, \\ 0 & \text { otherwise }\end{cases}
$$

In summary, we have defined a semi-Markov decision process with the state space $I$, action space $A$, sets $A(s)$ of available actions at states $s \in I$, transition probability $p\left(s, s^{\prime}, a\right)$, average sojourn time $\tau(s, a)$ in state $s \in I$ after action $a$ is chosen, reward function $r(s, a)$, and cost function $c(s, a)$.

Let $t_{0}=0$. If $t_{n}$ is defined for some $n=0,1, \ldots$, we define $t_{n+1}$ as the time epoch of either the next departure or the next arrival, whichever occurs first. Therefore, $0=t_{0}<t_{1}<\cdots$ is the order of the sequence of jump epochs, when the state of the system changes. A strategy $\pi$, which may be randomized and past-dependent, assigns actions $a_{n}$ at epoch $t_{n}$, to control the system. We define the long-run average rewards earned by the system as

$$
W(z, \pi)=\liminf _{t \rightarrow \infty} t^{-1} \mathrm{E}_{z}^{\pi} \sum_{n=0}^{N(t)-1} r\left(x_{n}, a_{n}\right)
$$


and the long-run average cost of the system as

$$
C(z, \pi)=\limsup _{t \rightarrow \infty} t^{-1} \mathrm{E}_{z}^{\pi} \sum_{n=0}^{N(t)-1} c\left(x_{n}, a_{n}\right),
$$

where $z$ is an initial state, $\pi$ is a strategy, $x_{n}$ is the state at epoch $t_{n}, \mathrm{E}_{z}^{\pi}$ is the expectation operator for the initial state $z$ and the strategy $\pi$, and $N(t)=\max \left\{n: t_{n} \leq t\right\}$ is the number of jumps made by time epoch $t$.

A strategy is called a randomized stationary policy if the assigned actions $a_{n}$ depend only on the current state $x_{n}$. In addition, if $a_{n}$ is a deterministic function of $x_{n}$, then the corresponding strategy is called a stationary policy.

According to [9, p. 471], the unichain condition holds for this model. The unichain condition requires that any randomized stationary policy define a Markov chain with one ergodic class and a (possibly empty) set of transient states on the system's state space. Under this condition, the objective functions $W(z, \phi)$ and $C(z, \phi)$ do not depend on the initial state $z \in I$ when $\phi$ is a randomized stationary policy. We shall therefore write $W(\phi)$ and $C(\phi)$ instead of $W(z, \phi)$ and $C(z, \phi)$, respectively, when $\phi$ is a randomized stationary policy. According to [5, Theorem 8.1(iv)], if the unichain condition holds and the semi-Markov decision problem is feasible for some $z$, then there exists a randomized stationary policy that is optimal for any initial state $z$, and the objective function does not depend on $z$. Thus, our problem can be modeled as the following optimization problem with a randomized stationary policy $\phi$ as the variable:

$$
\text { maximize } W(\phi) \text { subject to } C(\phi) \leq G, \quad G \in \mathbb{R} \text {. }
$$

Since an action can be chosen only at the arrival epochs, a randomized stationary policy $\phi$ for our problem can be defined by $\phi(n, i), n=0, \ldots, N-1, i=1, \ldots, m$, that is, the probability of accepting an arrival of type $i$ when there are $n$ customers in the system.

A randomized stationary policy $\phi$ is called $k$-randomized stationary, with $k=0,1,2, \ldots$, if the number of states $(n, i)$ with $0<\phi(n, i)<1$ is less than or equal to $k$. The notions of stationary and 0 -randomized stationary policies coincide.

Consider the following constraints for $(x, P)$, where

$$
x=\{x(n, i), n=0, \ldots, N-1, i=1, \ldots, m\}
$$

and $P=\left(P_{0}, \ldots, P_{N}\right)$ are respectively a matrix and a vector of real variables:

$$
\begin{gathered}
\sum_{i=1}^{m} \lambda_{i} c_{i}\left(1-\sum_{n=0}^{N-1} x(n, i)\right) \leq G, \\
\sum_{i=1}^{m} \lambda_{i} x(n, i)=\mu_{n+1} P_{n+1}, \quad n=0,1, \ldots, N-1, \\
\sum_{n=0}^{N} P_{n}=1, \\
n=0,1, \ldots, N-1, i=1,2, \ldots, m .
\end{gathered}
$$


For the same variables $x$ and $P$, we formulate the LP

$$
\operatorname{maximize} \sum_{i=1}^{m} \lambda_{i} r_{i} \sum_{n=0}^{N-1} x(n, i) \text { over } x \text { and } P \text {, subject to (2.2)-(2.5), }
$$

and the LP

$$
\text { maximize } \sum_{i=1}^{m} \lambda_{i} r_{i} \sum_{n=0}^{N-1} x(n, i) \text { over } x \text { and } P \text {, subject to (2.3)-(2.5). }
$$

For a vector $(x, P)$ satisfying (2.2)-(2.5), consider a randomized stationary policy $\phi$ such that

$$
\phi(n, i)= \begin{cases}x(n, i) / P_{n} & \text { if } P_{n}>0, n=0,1, \ldots, N-1, \text { and } i=1,2, \ldots, m, \\ \text { arbitrary } & \text { otherwise. }\end{cases}
$$

Theorem 2.1. (i) A randomized stationary policy $\phi$ is feasible for the problem (2.1) if and only if (2.8) holds for a feasible vector $(x, P)$ of the $L P(2.6)$.

(ii) If $(x, P)$ is an optimal solution to the $L P(2.6)$, then $P_{n}>0$ for all $n=0,1, \ldots, N$.

(iii) A randomized stationary policy $\phi$ is optimal for the problem (2.1) if and only if

$$
\phi(n, i)=x(n, i) / P_{n}, \quad n=0,1, \ldots, N-1, i=1,2, \ldots, m,
$$

holds for an optimal solution $(x, P)$ to the $L P(2.6)$. In addition, if $(x, P)$ is a basic optimal solution to the LP (2.6), then the policy $\phi$ defined in (2.9) is 1-randomized stationary optimal.

If $G \geq \sum_{i=1}^{m} \lambda_{i} c_{i}$, we face an unconstrained problem, namely

$$
\text { maximize } W(\phi) \text {, }
$$

and Theorem 2.1 implies the following result.

Corollary 2.1. (i) If $(x, P)$ is an optimal solution to the $L P(2.7)$, then $P_{n}>0$ for all $n=$ $0,1, \ldots, N$.

(ii) A randomized stationary policy $\phi$ is optimal for the problem (2.10) if and only if (2.9) holds for an optimal solution $(x, P)$ to the LP (2.7). In addition, if $(x, P)$ is a basic optimal solution to the LP (2.7), then the policy $\phi$ defined in (2.9) is nonrandomized stationary optimal.

In view of (2.4) and (2.5), the feasible region of the LP (2.6) is bounded. Therefore, this LP has an optimal solution, if it is feasible. If the LP (2.6) is feasible, we consider an arbitrary optimal dual solution $(\bar{u}, \bar{v})$, with $\bar{u}=\left(\bar{u}_{1}, \ldots, \bar{u}_{2 m N+1}\right)$ and $\bar{v}=\left(\bar{v}_{1}, \ldots, \bar{v}_{N+1}\right)$, where $\bar{u}$ corresponds to all inequality constraints and $\bar{v}$ corresponds to equality constraints, and introduce the following LP:

$$
\operatorname{maximize} \sum_{i=1}^{m} \lambda_{i}\left(r_{i}+\bar{u}_{1} c_{i}\right) \sum_{n=0}^{N-1} x(n, i)-\bar{u}_{1}\left(\sum_{i=1}^{m} \lambda_{i} c_{i}-G\right) \text { over } x \text { and } P
$$


Notice that most of the contemporary LP solvers use interior-point methods and calculate the primary and dual solutions simultaneously. Therefore, we do not formulate the dual LP in this paper. Here $\bar{u}_{1}$ is also called the Lagrange multiplier with respect to the first constraint. More details about the Lagrangian function and Lagrange multipliers can be found in Appendix B. Lemma B.1 in Appendix B, and the explanations preceding it, imply the following result.

Lemma 2.1. If the $L P(2.6)$ is feasible then (i) any optimal solution to the $L P(2.6)$ is an optimal solution to the LP (2.11), and (ii) the optimal values of the objective functions for these two LPs are equal.

We notice that, for any randomized stationary policy $\phi$, there is a unique solution $P^{\phi}$ to the following birth-and-death equations:

$$
\begin{aligned}
\sum_{i=1}^{m} \lambda_{i} \phi(n, i) P_{n} & =\mu_{n+1} P_{n+1}, \quad n=0,1, \ldots, N-1, \\
\sum_{n=0}^{N} P_{n} & =1 .
\end{aligned}
$$

Here $\phi$ is defined by $\phi(n, i), n=0, \ldots, N-1, i=1, \ldots, m$, the probability of accepting an arrival of type $i$ when there are $n$ customers in the system, and $P_{n}^{\phi}$ is the limiting probability that there are $n$ customers in the system when the randomized stationary policy $\phi$ is used.

In addition, we define

$$
x^{\phi}(n, i)=\phi(n, i) P_{n}^{\phi}, \quad n=0,1, \ldots, N-1, i=1,2, \ldots, m .
$$

Then $\left(x^{\phi}, P^{\phi}\right)$ satisfies (2.3)-(2.5) and is therefore a feasible solution to the LP (2.7). In view of Theorem 2.1(i), a randomized stationary policy $\phi$ is feasible for the problem (2.1) if and only if $\left(x^{\phi}, P^{\phi}\right)$ is a feasible solution to the LP (2.6). In addition, according to Theorem 2.1(iii), a randomized stationary policy is optimal for the problem $(2.1)$ if and only if $\left(x^{\phi}, P^{\phi}\right)$ is optimal for the LP (2.6). In particular, according to Corollary 2.1, a randomized stationary policy $\phi$ is optimal for the unconstrained problem (2.10) if and only if the vector $\left(x^{\phi}, P^{\phi}\right)$ is optimal for the LP (2.7).

The following theorem geometrically links the optimal solutions to the LP (2.6) to feasible vectors for the LP (2.7).

Theorem 2.2. Let $\phi$ be a 1-randomized stationary optimal policy for the problem (2.1). If there exists a state $\left(n_{0}, i_{0}\right)$ with $0<\phi\left(n_{0}, i_{0}\right)<1$, consider two stationary policies, $\phi^{\prime}$ and $\phi^{\prime \prime}$, that coincide with $\phi$ at all states except for the state $\left(n_{0}, i_{0}\right)$ and satisfy $\phi^{\prime}\left(n_{0}, i_{0}\right)=0$ and $\phi^{\prime \prime}\left(n_{0}, i_{0}\right)=1$. Then, for some $\alpha, 0<\alpha<1$,

$$
\left(x^{\phi}, P^{\phi}\right)=\alpha\left(x^{\phi^{\prime}}, P^{\phi^{\prime}}\right)+(1-\alpha)\left(x^{\phi^{\prime \prime}}, P^{\phi^{\prime \prime}}\right) .
$$

The following theorem is the main result of this paper.

Theorem 2.3. Any 1-randomized stationary optimal policy for the problem (2.1) is a randomized trunk reservation policy that is consistent with the reward function $r_{i}^{\prime}=r_{i}+\bar{u}_{1} c_{i}$, $i=1, \ldots, m$, where $\bar{u}_{1} \geq 0$ is the Lagrange multiplier with respect to the constraint (2.2).

Consider an average-reward semi-Markov decision process with one constraint. If the unichain condition holds and a feasible policy exists, then there exists a 1-randomized stationary optimal policy [5]. Therefore, the previous theorem implies the following corollary. 
Corollary 2.2. If the problem (2.1) is feasible, then there exists an optimal randomized trunk reservation policy that is consistent with $r^{\prime}$.

Let

$$
c_{i}= \begin{cases}1 / \lambda_{j}, & i=j, \\ 0, & \text { otherwise. }\end{cases}
$$

According to $\left[9\right.$, p. 471], for the costs $c_{i}$ defined by $(2.15)$, the average cost $C(z, \pi)$ is the blocking probability for type- $j$ customers. Therefore, the problem of maximizing the average rewards per unit time subject to the constraint that the blocking probability for type- $j$ customers does not exceed $q$ is equivalent to the problem (2.1) with the cost function $c$ defined in (2.15).

The following corollary describes the structure of optimal policies when the objective is to maximize the average rewards per unit time subject to the constraint on the blocking probability for type- $j$ customers.

Corollary 2.3. Consider a special case of problem (2.1) with the constraint on the blocking probability of type- $j$ customers, $j=1, \ldots, m$. If this problem is feasible then any 1 -randomized stationary optimal policy is a randomized trunk reservation policy consistent with the reward function $r^{\prime}$ that was defined in (1.1) and has the properties that $r_{i}^{\prime}=r_{i}$ if $i \neq j$ and $r_{j}^{\prime} \geq r_{j}$.

In particular, when $j=1$, Corollary 2.3 implies the following statement.

Corollary 2.4. Consider a special case of problem (2.1) with the constraint on the blocking probability of the most profitable customers (those of type 1). If this problem is feasible then any 1-randomized stationary optimal policy is a randomized trunk reservation policy consistent with the rewards $r_{i}$.

In particular, for the case in which $r_{1}>r_{2}>\cdots>r_{m}$, Corollary 2.4 coincides with the main result of [9]. If the cost constraint limits the blocking probability for several customer types pooled together, say for customer types belonging to a set $J, J \subset\{1, \ldots, m\}$, then we define $\Lambda_{J}=\sum_{j \in J} \lambda_{j}$ and

$$
c_{i}= \begin{cases}1 / \Lambda_{J} & \text { if } j \in J, \\ 0 & \text { otherwise }\end{cases}
$$

Then the combined blocking probability for customers in the set $J$ under policy $\pi$ and with initial state $z$ is $C(z, \pi)$, with the function $c_{i}$ defined by (2.16).

The following corollary describes the structure of optimal policies when the objective is to maximize the average rewards per unit time subject to the constraint on the combined blocking probability for several customer types.

Corollary 2.5. Consider a special case of problem (2.1) with the constraint on the combined blocking probability for customer types belonging to a set $J, J \subset\{1, \ldots, m\}$. If this problem is feasible then any 1-randomized stationary optimal policy is a randomized trunk reservation policy consistent with a function $r^{\prime}$, where

$$
r_{i}^{\prime}= \begin{cases}r_{i}+\bar{u}_{1} / \Lambda_{J} & \text { if } i \in J \\ r_{i} & \text { otherwise }\end{cases}
$$

and (i) $r_{i}^{\prime}=r_{i}$ if $i \notin J$, (ii) $r_{i}^{\prime} \geq r_{i}$ if $i \in J$, and (iii) $r_{i}^{\prime} \geq r_{j}^{\prime}$ if $i, j \in J$ and $r_{i} \geq r_{j}$. 


\section{Unconstrained problem}

For an $\mathrm{M} / \mathrm{M} / c /$ loss system, Miller [21] proved that if $r_{1}>r_{2}>\cdots>r_{m}>0$, then there exists a trunk reservation optimal policy, consistent with the rewards $r_{i}$, for the unconstrained problem (2.10). Feinberg and Reiman [9] proved the following stronger result.

Lemma 3.1. ([9, Corollary 3.7].) If $r_{1}>\cdots>r_{m}>0$ then any (nonrandomized) stationary optimal policy for the unconstrained problem (2.10) is a trunk reservation policy consistent with the rewards $r_{i}, i=1, \ldots, m$.

The next lemma is a particular case of Lemma 2.5 of [9], which dealt with constrained problems in which the constraint was that the blocking probability of type- 1 customers be no greater than $q$. By setting $q=1$ in the mentioned results of [9], we obtain the following result for the unconstrained problem.

Lemma 3.2. A randomized stationary policy $\phi$ is optimal for the problem (2.10) if and only if $\phi(n, i)$ and a vector $P=\left(P_{0}, \ldots, P_{n}\right)$ form an optimal solution to the following nonlinear program:

$$
\begin{aligned}
& \text { maximize } \sum_{i=1}^{m} \lambda_{i} r_{i}\left(\sum_{n=0}^{N-1} \phi(n, i) P_{n}\right) \text { over } x \text { and } P \text {, subject to } \\
& \qquad \leq \leq(n, i) \leq 1, n=0,1, \ldots, N-1, i=1,2, \ldots, m \text {, and (2.12) and (2.13). }
\end{aligned}
$$

We remark that, according to Lemma 3.1, if $r_{1}>\cdots>r_{m}>0$ then any stationary optimal policy has a trunk reservation form and $N-1=M_{1} \geq \cdots \geq M_{m}$. The following example shows that the optimal thresholds $M_{1}, \ldots, M_{m}$ may not be unique.

Example 3.1. Consider an $M / M / 1 /$ loss system with two types of customer, with $\lambda_{1}=\lambda_{2}=$ $\mu=1, r_{1}=2$, and $r_{2}=1$. In this example, an arrival can be accepted only when the system is empty, and there are only two trunk reservation policies consistent with the rewards $r_{1}$ and $r_{2}$ : (i) accept all the arrivals, and (ii) accept only type-1 arrivals. According to Lemma 3.1, at least one of these two policies is optimal. Straightforward analysis of the birth-and-death process defined by (2.12) and (2.13), with two states, yields $P_{0}=\frac{1}{3}$ and $P_{1}=\frac{2}{3}$, with an expected average reward per unit time, computed as in the expression to be maximized in (3.1), equal to 1 , for the first policy. For the second policy, $P_{0}=P_{1}=\frac{1}{2}$ and the expected average reward per unit time also equals 1 . Thus, trunk reservation policies (i) and (ii) are both optimal. Moreover, consider a randomized trunk reservation policy that, when the system is empty, always accepts type- 1 arrivals and accepts type- 2 arrivals independently with some probability, say $p$. Under this policy, straightforward calculations for the birth-and-death process yield $P_{0}=1 /(2+p)$ and $P_{1}=(1+p) /(2+p)$. Therefore, the expected average rewards per unit time equal $2 \times 1 /(2+p)+p \times 1 /(2+p)=1$, for any randomized trunk reservation policy.

The following lemma covers the case in which $r_{1} \geq \cdots \geq r_{m}>0$. However, being motivated by constrained problems, for which it is possible that $r_{i}^{\prime}<r_{i+1}^{\prime}$, we do not specify these inequalities in the lemma. Since its proof is rather technical, we present it in Appendix A.

Lemma 3.3. Consider any randomized stationary optimal policy $\phi$ for the unconstrained problem (2.10).

(i) For any $i$ and $j$ such that $r_{i}>r_{j}$, we have

$$
\phi(n, i) \geq \phi(n, j), \quad n=0, \ldots, N-1, i, j=1, \ldots, m .
$$


(ii) For each $n=0, \ldots, N-1$, if there exist two customer types, $j_{1}$ and $j_{2}$, such that $0<\phi(n, j)<1, j=j_{1}, j_{2}$, then $r_{j_{1}}=r_{j_{2}}$. In particular, if the rewards $r_{1}, \ldots, r_{m}$ are all different, then, for each $n=0, \ldots, N-1$, the probabilities $\phi(n, j), j=1, \ldots, m$, except for at most one of them, are all equal to either 0 or 1 .

(iii) There exists at least one customer type, say type $\ell$, such that

$$
\phi(n, \ell)=1, \quad n=0, \ldots, N-1 .
$$

In particular, if $r_{j}=\max \left\{r_{i}, i=1, \ldots, m\right\}$ then (3.2) holds with $\ell=j$.

(iv) We have

$$
\phi(n, j) \geq \phi(n+1, j), \quad n=0, \ldots, N-2, j=1, \ldots, m,
$$

and, for each $j=1, \ldots, m$, the probabilities $\phi(n, j), n=0, \ldots, N-1$, except for at most one of them, are all equal to either 0 or 1 .

The following corollary of Lemma 3.3 is used in the proof of Theorem 2.3.

Corollary 3.1. Any stationary optimal policy $\varphi$ for the unconstrained problem (2.10) is a trunk reservation policy consistent with the rewards $r_{i}$.

The following example demonstrates that if different types of customer have the same rewards, then, for an optimal trunk reservation policy $\phi$, the existence of which follows from Corollary 3.1, it is possible that $M_{i}^{\phi}<M_{i+1}^{\phi}$ when $r_{i}=r_{i+1}, i=1, \ldots, m-1$.

Example 3.2. We consider Example 3.1 and split type-2 customers into types 2 and 3, with $\lambda_{2}=0.4$ and $\lambda_{3}=0.6$. We then have $\lambda_{1}=\mu=1, \lambda_{2}=0.4, \lambda_{3}=0.6, r_{1}=2$, and $r_{2}=r_{3}=1$, and the trunk reservation policy $\phi$ with $\left(M_{1}^{\phi}, M_{2}^{\phi}, M_{3}^{\phi}\right)=(1,0,1)$ is optimal and $W(\phi)=1$. Although $r_{1}>r_{2}=r_{3}$, it is not necessary that $M_{1}^{\phi} \geq M_{2}^{\phi} \geq M_{3}^{\phi}$.

The following property of an optimal solution to the LP (2.7) is essential to the proof of Theorem 2.1.

Lemma 3.4. If $(x, P)$ is an optimal solution to (2.7), then $P_{n}>0$ for all $n=0,1, \ldots, N$.

Proof. The proof is based on contradiction. Without loss of generality, let us assume that $r_{1} \geq r_{2} \geq \cdots \geq r_{m}>0$. Let $(x, P)$ be an optimal solution to the LP (2.7) such that there exists an $n^{*}, 0<n^{*} \leq N$, for which $P_{n^{*}}=0$. Then, by (2.4) and (2.5) there exists an $n_{0}, 0 \leq n_{0}<N$, for which $P_{n_{0}}>0$ and $P_{n_{0}+1}=0$. In view of (2.3) and (2.5) we have (i) $x(n, i)=0$ for $n \geq n_{0}$ and $i=1, \ldots, m$, (ii) $P_{n}=0$ for $n>n_{0}$, and (iii) $P_{n}>0$ for $n \leq n_{0}$. In the following we construct a feasible point $\left(x^{\prime}, P^{\prime}\right)$ that achieves a larger value of the objective function than does $(x, P)$. We define

$$
\begin{aligned}
P_{n}^{\prime}= \begin{cases}\frac{\mu_{n_{0}+1}}{\mu_{n_{0}+1}+\lambda_{1} P_{n_{0}}} P_{n}, & n \leq n_{0}, \\
\frac{\lambda_{1}}{\mu_{n_{0}+1}+\lambda_{1} P_{n_{0}}} P_{n_{0}}, & n=n_{0}+1, \\
0, & \text { otherwise, }\end{cases} \\
x^{\prime}(n, i)= \begin{cases}\left(1-P_{n_{0}+1}^{\prime}\right) x(n, i), & n<n_{0}, i=0, \ldots, m, \\
P_{n_{0}}^{\prime}, & n=n_{0}, i=1, \\
0, & \text { otherwise. }\end{cases}
\end{aligned}
$$


We observe that $\left(x^{\prime}, P^{\prime}\right)$ satisfies (2.3)-(2.5). Indeed, (2.4) and (3.3) imply that

$$
P_{n}^{\prime}=\left(1-P_{n_{0}+1}^{\prime}\right) P_{n}, \quad n \leq n_{0}, \quad P_{n_{0}+1}^{\prime}=P_{n_{0}}^{\prime} \frac{\lambda_{1}}{\mu_{n_{0}+1}} .
$$

These equalities and (3.4) imply, after some simple algebra, that the vector $\left(x^{\prime}, P^{\prime}\right)$ satisfies (2.3) and (2.5). To verify (2.4), note that

$$
\begin{aligned}
\sum_{n=1}^{N} P_{n}^{\prime}=\sum_{n=1}^{n_{0}+1} P_{n}^{\prime} & =\sum_{n=1}^{n_{0}} \frac{\mu_{n_{0}+1} P_{n}}{\mu_{n_{0}+1}+\lambda_{1} P_{n_{0}}}+\frac{\lambda_{1} P_{n_{0}}}{\mu_{n_{0}+1}+\lambda_{1} P_{n_{0}}} \\
& =\frac{\mu_{n_{0}+1}}{\mu_{n_{0}+1}+\lambda_{1} P_{n_{0}}}+\frac{\lambda_{1} P_{n_{0}}}{\mu_{n_{0}+1}+\lambda_{1} P_{n_{0}}} \\
& =1,
\end{aligned}
$$

where the first two equalities follow from (3.3) and the third follows from $\sum_{n=1}^{n_{0}} P_{n}=1$.

Denote the values of the objective function at $(x, P)$ and $\left(x^{\prime}, P^{\prime}\right)$ by $W$ and $W^{\prime}$, respectively. In the following, we prove that $W^{\prime}>W$. Notice that

$$
W^{\prime}=\left(1-P_{n_{0}+1}^{\prime}\right) W+r_{1} \lambda_{1} P_{n_{0}}^{\prime}=\left(1-P_{n_{0}+1}^{\prime}\right) W+r_{1} \mu_{n_{0}+1} P_{n_{0}+1}^{\prime},
$$

where the first equality follows from (3.4) and the second follows from (3.3). By rearranging (3.5), we obtain

$$
W^{\prime}-W=P_{n_{0}+1}^{\prime}\left(r_{1} \mu_{n_{0}+1}-W\right) .
$$

In addition,

$$
W=\sum_{i=1}^{m} \lambda_{i} r_{i} \sum_{n=0}^{n_{0}-1} x(n, i) \leq r_{1} \sum_{n=0}^{n_{0}-1} \sum_{i=1}^{m} \lambda_{i} x(n, i)=r_{1} \sum_{n=0}^{n_{0}-1} \mu_{n+1} P_{n+1} \leq r_{1} \mu_{n_{0}} \sum_{n=0}^{n_{0}-1} P_{n+1},
$$

where the first equality follows from $x(n, i)=0, n \geq n_{0}, i=1, \ldots, m$, the first inequality follows from the assumption that $r_{1} \geq r_{2} \geq \cdots \geq r_{m}$, the second equality follows from (2.4), and the last inequality follows from the assumption that $\mu_{1} \leq \mu_{2} \leq \cdots \leq \mu_{n}$. Therefore,

$$
W \leq r_{1} \mu_{n_{0}} \sum_{n=1}^{n_{0}} P_{n}<r_{1} \mu_{n_{0}} \sum_{n=0}^{n_{0}} P_{n}=r_{1} \mu_{n_{0}} \leq r_{1} \mu_{n_{0}+1},
$$

where the strict inequality follows from $P_{0}>0$. Since $P_{n_{0}+1}^{\prime}>0$, we see that $W^{\prime}-W>0$. Note that the value of the objective function is strictly greater at $\left(x^{\phi^{\prime}}, P^{\phi^{\prime}}\right)$ than it is at $\left(x^{\phi}, P^{\phi}\right)$, and that $\left(x^{\phi^{\prime}}, P^{\phi^{\prime}}\right)$ satisfies (2.3)-(2.5). Therefore, $(x, P)$ is not optimal. This contradiction completes the proof.

\section{Justification of the LP formulation}

Lemma 3.2 established a link between the unconstrained problem (2.10) and the nonlinear program (3.1). According to the following lemma, the constraint in (2.1) adds an equation to the system (3.1). Based on this, we are ready to give the proof of Theorem 2.1. Notice that, for any randomized stationary policy $\phi$, there exists a unique vector $\left(P_{1}, \ldots, P_{n}\right)$ satisfying $(2.12)$ and (2.13). 
Lemma 4.1. A randomized stationary policy $\phi$ is optimal (or feasible) for the problem (2.1) if and only if $\phi(n, i)$ and the vector $P^{\phi}=\left(P_{0}^{\phi}, \ldots, P_{N}^{\phi}\right)$, respectively defined by (2.12) and (2.13), form an optimal (feasible) solution to the nonlinear program

$$
\begin{aligned}
& \text { maximize } \sum_{i=1}^{m} \lambda_{i} r_{i}\left(\sum_{n=0}^{N-1} \phi(n, i) P_{n}\right) \text { over } \phi \text { and } P, \text { subject to } \\
& \sum_{i=1}^{m} \lambda_{i} c_{i}\left(1-\sum_{n=0}^{N-1} \phi(n, i) P_{n}\right) \leq G, \\
& 0 \leq \phi(n, i) \leq 1, \quad n=0,1, \ldots, N-1, i=1,2, \ldots, m, \\
& \text { (2.12) and (2.13). }
\end{aligned}
$$

Proof. The proof is similar to the proof of Lemma 2.5 of [9].

Proof of Theorem 2.1. (i) Consider a randomized stationary policy $\phi$ feasible for the problem (2.1). Lemma 4.1 implies that the vector $P^{\phi}$ defined by (2.12) and (2.13) is feasible for the nonlinear program (4.1). The function $x^{\phi}$, defined by (2.14), and the vector $P^{\phi}$ satisfy (2.2)-(2.5). In addition, (2.14) implies (2.8). Conversely, consider any feasible vector $(x, P)$ for the LP (2.6), and let $\phi(n, i)$ be a randomized stationary policy satisfying (2.8). Then

$$
x(n, i)= \begin{cases}\phi(n, i) P_{n} & \text { if } P_{n}>0, n=0,1, \ldots, N-1, \text { and } i=1,2, \ldots, m, \\ 0 & \text { otherwise. }\end{cases}
$$

Therefore, $\left(\phi(n, i), P_{n}\right)$ is feasible for the nonlinear program (4.1). In view of Lemma 4.1, $\phi$ is a randomized stationary policy feasible for the problem $(2.1)$.

(ii) Consider the LP (2.11). Lemma 3.4 implies that $P_{n}>0, n=0, \ldots, N$, for any optimal solution $(x, P)$ to this LP. By Lemma 2.1, any optimal solution $(x, P)$ to the $\operatorname{LP}(2.6)$ is optimal for the LP (2.11).

(iii) Consider a randomized stationary optimal policy $\phi$ for the problem (2.1). In view of Theorem 2.1(i), $\left(x^{\phi}, P^{\phi}\right)$, defined by (2.12)-(2.14), is a feasible solution to the LP (2.6). According to Lemma 4.1, $\left(\phi, P^{\phi}\right)$ is an optimal solution to the nonlinear program (4.1). We shall prove by contradiction that $\left(x^{\phi}, P^{\phi}\right)$ is an optimal solution to the LP (2.6).

Since the feasible region of the LP (2.6) is bounded, there exists an optimal solution $\left(x^{\prime}, P^{\prime}\right)$ to this LP. Suppose that $\left(x^{\prime}, P^{\prime}\right)$ achieves a larger objective value than does $\left(x^{\phi}, P^{\phi}\right)$. Due to Theorem 2.1(ii), $P_{n}^{\prime}>0$ for all $n=0, \ldots, N$. Let us define $\phi^{\prime}(n, i)=x^{\prime}(n, i) / P_{n}^{\prime}$. Then $\left(\phi^{\prime}, P^{\prime}\right)$ is feasible for the LP (4.1) and achieves a larger value of the objective function than does $\left(\phi, P^{\phi}\right)$. This contradicts the fact that $\left(\phi, P^{\phi}\right)$ is an optimal solution to (4.1).

Conversely, let $(x, P)$ be an optimal solution to the LP (2.6). We define

$$
\phi(n, i)=\frac{x(n, i)}{P_{n}}, \quad n=0, \ldots, N, i=1, \ldots, m .
$$

According to Lemma 4.1, it suffices to prove that $(\phi, P)$ is an optimal solution to the nonlinear program (4.1), and we shall use contradiction to do so. Suppose that there exists a feasible solution $\left(\phi^{\prime}, P^{\prime}\right)$ to $(4.1)$ which achieves a larger objective value than does $(\phi, P)$. Let $x^{\prime}(n, i)=$ $\phi^{\prime}(n, i) P_{n}^{\prime}$. Then $\left(x^{\prime}, P^{\prime}\right)$ is feasible for the LP (2.6), and achieves a larger value of the objective function than does $(x, P)$. This contradicts the fact that $(x, P)$ is optimal for the LP (2.6). 
To prove the second statement in (iii), first we note that the first statement of Theorem 2.1(iii) (proved above) implies that $\phi$ is optimal. Second, we represent the LP (2.6) in a standard LP form, in which the nonnegative variable $S$ is introduced to replace (2.2) with

$$
\sum_{i=1}^{m} \lambda_{i} c_{i}\left(1-\sum_{n=0}^{N-1} x(n, i)\right)+S=G
$$

and nonnegative variables $y(n, i), n=0, \ldots, N-1, i=1, \ldots, m$, are introduced to replace (2.5) with $x(n, i)+y(n, i)=P_{n}$. There are $2+N+N \times m$ constraints and $N+2+2(N \times m)$ variables for this new LP. Therefore, any basic optimal solution to this new LP has at most $2+N+N \times m$ basic variables. Since $P_{n}, n=0, \ldots, N$, are positive, there are at most $1+N \times m$ basic variables among $x(n, i)$ and $y(n, i)$. Because $x(n, i)+y(n, i)=P_{n}>0$, $x(n, i)$ and $y(n, i)$ cannot be 0 simultaneously. Therefore, for each pair $(n, i)$, either $x(n, i)=0$ or $y(n, i)=0$, except for at most one pair, for which both of them are nonzero. Since $\phi(n, i)=x(n, i) / P_{n}$, we find that for all but at most one of the pairs $(n, i), \phi(n, i)$ equals either 0 or 1 . Therefore, the policy $\phi$ is 1 -randomized stationary optimal.

Proof of Corollary 2.1. Statement (i) is Lemma 3.4. Also, (i) and the first part of (ii) follow from Theorem 2.1(ii) and (iii), respectively, when $G \geq 1$. The proof of the second part of statement (ii) is nearly identical to the proof of the second part of Theorem 2.1(iii), the only difference being that there are $1+N+N \times m$ constraints and, therefore, there are at most $N \times m$ basic variables among $x(n, i)$ and $y(n, i)$. Hence, for each pair $(n, i)$, either $x(n, i)=0$ or $y(n, i)=0$, but they do not hold simultaneously. This gives us a nonrandomized policy $\phi$.

\section{Geometric properties of optimal solutions}

In this section, we prove Theorem 2.2. Consider policies $\phi, \phi^{\prime}$, and $\phi^{\prime \prime}$ as in Theorem 2.2. According to Theorem 2.1(iii), $\left(x^{\phi}, P^{\phi}\right)$ is an optimal solution to the LP (2.6). In addition, both $\left(x^{\phi^{\prime}}, P^{\phi^{\prime}}\right)$ and $\left(x^{\phi^{\prime \prime}}, P^{\phi^{\prime \prime}}\right)$ are feasible solutions to the LP (2.7).

Lemma 5.1. Let the policies $\phi, \phi^{\prime}$, and $\phi^{\prime \prime}$ be defined as in Theorem 2.2, and consider the vectors $P^{\phi}, P^{\phi^{\prime}}$, and $P^{\phi^{\prime \prime}}$ defined by (2.12) and (2.13). The following expressions hold:

$$
\begin{gathered}
\frac{P_{0}^{\phi^{\prime}}}{P_{0}^{\phi}}=\frac{P_{1}^{\phi^{\prime}}}{P_{1}^{\phi}}=\cdots=\frac{P_{n_{0}}^{\phi^{\prime}}}{P_{n_{0}}^{\phi}}, \\
\frac{P_{n_{0}+1}^{\phi^{\prime}}}{P_{n_{0}+1}^{\phi}}=\frac{P_{n_{0}+2}^{\phi^{\prime}}}{P_{n_{0}+2}^{\phi}}=\cdots=\frac{P_{N}^{\phi^{\prime}}}{P_{N}^{\phi}}, \\
\frac{P_{0}^{\phi^{\prime \prime}}}{P_{0}^{\phi}}=\frac{P_{1}^{\phi^{\prime \prime}}}{P_{1}^{\phi}}=\cdots=\frac{P_{n_{0}}^{\phi^{\prime \prime}}}{P_{n_{0}}^{\phi}}, \\
\frac{P_{n_{0}+1}^{\phi^{\prime \prime}}}{P_{n_{0}+1}^{\phi}}=\frac{P_{n_{0}+2}^{\phi^{\prime \prime}}}{P_{n_{0}+2}^{\phi}}=\cdots=\frac{P_{N}^{\phi^{\prime \prime}}}{P_{N}^{\phi}} .
\end{gathered}
$$

In addition, $t_{1}, t_{2}, t_{3}$, and $t_{4}$, the respective values of the ratios in (5.1)-(5.4), satisfy $t_{1}>1$, $t_{2}<1, t_{3}<1$, and $t_{4}>1$. 
Proof. From (2.12), it follows that

$$
\begin{aligned}
& \sum_{i=1}^{m} \lambda_{i} \phi^{\prime}(n, i) P_{n}^{\phi^{\prime}}=\mu_{n+1} P_{n+1}^{\phi^{\prime}}, \quad n=0,1, \ldots, N-1, \\
& \sum_{i=1}^{m} \lambda_{i} \phi(n, i) P_{n}^{\phi}=\mu_{n+1} P_{n+1}^{\phi}, \quad n=0,1, \ldots, N-1 .
\end{aligned}
$$

Notice that $\phi(n, i)=\phi^{\prime}(n, i)$ when $(n, i) \neq\left(n_{0}, i_{0}\right), n=0, \ldots, N-1, i=1, \ldots, m$, and that $P_{n}^{\phi}>0, n=0, \ldots, N$, according to Theorem 2.1(ii). Therefore, we can divide (5.5) by (5.6), to obtain

$$
\frac{P_{n}^{\phi^{\prime}}}{P_{n}^{\phi}}=\frac{P_{n+1}^{\phi^{\prime}}}{P_{n+1}^{\phi}}, \quad n=0, \ldots, N-1, n \neq n_{0},
$$

which is equivalent to (5.1) and (5.2) simultaneously. The proof of (5.3) and (5.4) is similar.

Since $\phi^{\prime}\left(n_{0}, i_{0}\right)=0$ and $\phi^{\prime \prime}\left(n_{0}, i_{0}\right)=1$, we have

$$
\sum_{i=1}^{m} \phi^{\prime}\left(n_{0}, i\right) \lambda_{i}=\sum_{i=1}^{m} \phi\left(n_{0}, i\right) \lambda_{i}-\lambda_{i_{0}} \phi\left(n_{0}, i_{0}\right)
$$

and

$$
\sum_{i=1}^{m} \phi^{\prime \prime}\left(n_{0}, i\right) \lambda_{i}=\sum_{i=1}^{m} \phi\left(n_{0}, i\right) \lambda_{i}+\lambda_{i_{0}}\left(1-\phi\left(n_{0}, i_{0}\right)\right) .
$$

Then

$$
P_{n_{0}+1}^{\phi^{\prime}}=\sum_{i=1}^{m} \phi^{\prime}\left(n_{0}, i\right) \lambda_{i} \frac{P_{n_{0}}^{\phi^{\prime}}}{\mu_{n_{0}+1}}=\left(\frac{\mu_{n_{0}+1} P_{n_{0}+1}^{\phi}}{P_{n_{0}}^{\phi}}-\lambda_{i_{0}} \phi\left(n_{0}, i_{0}\right)\right) \frac{P_{n_{0}}^{\phi^{\prime}}}{\mu_{n_{0}+1}},
$$

where the first equality follows from (2.12) and the second equality follows from (5.7) and from (2.12), applied sequentially. Similarly,

$$
P_{n_{0}+1}^{\phi^{\prime \prime}}=\left(\frac{\mu_{n_{0}+1} P_{n_{0}+1}^{\phi}}{P_{n_{0}}^{\phi}}+\lambda_{i_{0}}\left(1-\phi\left(n_{0}, i_{0}\right)\right)\right) \frac{P_{n_{0}}^{\phi^{\prime \prime}}}{\mu_{n_{0}+1}} .
$$

By dividing the left-most and right-most expressions in (5.8) by $P_{n_{0}+1}^{\phi}$, we obtain

$$
\frac{P_{n_{0}+1}^{\phi^{\prime}}}{P_{n_{0}+1}^{\phi}}=\frac{P_{n_{0}}^{\phi^{\prime}}}{P_{n_{0}}^{\phi}}-\frac{\lambda_{i_{0}} \phi\left(n_{0}, i_{0}\right) P_{n_{0}}^{\phi^{\prime}}}{\mu_{n_{0}+1} P_{n_{0}+1}^{\phi}} .
$$

We observe that $P_{n_{0}}^{\phi^{\prime}}>0$. Indeed, if $P_{n_{0}}^{\phi^{\prime}}=0$ then (5.8) implies that $P_{n_{0}+1}^{\phi^{\prime}}=0$. In view of (5.1) and (5.2), $P_{n}^{\phi^{\prime}}=0$ for all $n=0, \ldots, N$. Therefore, $P^{\phi^{\prime}}$ is not a probability vector.

Now we shall prove that $t_{1}>1$, by contradiction. Suppose that $t_{1} \leq 1$. Then (5.1) implies that $P_{n}^{\phi^{\prime}} \leq P_{n}^{\phi}$ when $n \leq n_{0}$. In view of (5.2) and (5.10), we have $P_{n}^{\phi^{\prime}}<P_{n}^{\phi}$ for $n>n_{0}$. Therefore, $\sum_{i=0}^{N} P_{i}^{\phi^{\prime}}<\sum_{i=0}^{N} P_{i}^{\phi}=1$, which contradicts the fact that $P^{\phi^{\prime}}$ is a probability vector. 
Next we prove that $t_{2}<1$. Since $t_{1}>1$, from (5.1) we have $P_{n}^{\phi^{\prime}}>P_{n}^{\phi}$ for $n \leq n_{0}$. Suppose that $t_{2} \geq 1$. Then $P_{n}^{\dot{\phi}^{\prime}} \geq P_{n}^{\phi}$ for $n>n_{0}$. Thus, $\sum_{i=0}^{N} P_{i}^{\phi^{\prime}}>\sum_{i=0}^{N} P_{i}^{\phi}=1$. This is a contradiction, proving that $t_{2}<1$.

To prove that $t_{3}<1$ and $t_{4}>1$, we divide both sides of (5.9) by $P_{n_{0}+1}^{\phi}$, to obtain

$$
\frac{P_{n_{0}+1}^{\phi^{\prime \prime}}}{P_{n_{0}+1}^{\phi}}=\frac{P_{n_{0}}^{\phi^{\prime \prime}}}{P_{n_{0}}^{\phi}}+\frac{\lambda_{i_{0}}\left(1-\phi\left(n_{0}, i_{0}\right)\right) P_{n_{0}}^{\phi^{\prime \prime}}}{\mu_{n_{0}+1} P_{n_{0}+1}^{\phi}} .
$$

The rest of the proof is similar to the proofs that $t_{1}>1$ and $t_{2}<1$.

Lemma 5.2. Consider the policy $\phi$ described in Theorem 2.2, and let $t_{1}, t_{2}, t_{3}$, and $t_{4}$ be the constants defined in Lemma 5.1. Then

$$
\frac{1-t_{2}}{t_{4}-t_{2}}=\frac{t_{1}-1}{t_{1}-t_{3}}=\frac{\phi\left(n_{0}, i_{0}\right)}{t_{3}} .
$$

Proof. In view of (5.1) and (5.2), we have

$$
t_{1} \sum_{i=0}^{n_{0}} P_{i}^{\phi}+t_{2} \sum_{i=n_{0}+1}^{N} P_{i}^{\phi}=\sum_{i=0}^{N} P_{i}^{\phi^{\prime}}=1 .
$$

Similarly, (5.3) and (5.4) imply that

$$
t_{3} \sum_{i=0}^{n_{0}} P_{i}^{\phi}+t_{4} \sum_{i=n_{0}+1}^{N} P_{i}^{\phi}=1 .
$$

We introduce $u=\sum_{i=0}^{n_{0}} P_{i}^{\phi}$ and $v=\sum_{i=n_{0}+1}^{N} P_{i}^{\phi}$. Then (5.13) and (2.13) can be rewritten as

$$
\begin{aligned}
t_{1} u+t_{2} v & =1, \\
u+v & =1 .
\end{aligned}
$$

This implies that

$$
v=\frac{t_{1}-1}{t_{1}-t_{2}}
$$

Similarly, (5.14) and (2.13) imply that

$$
v=\frac{1-t_{3}}{t_{4}-t_{3}}
$$

Thus,

$$
v=\frac{t_{1}-1}{t_{1}-t_{2}}=\frac{1-t_{3}}{t_{4}-t_{3}} .
$$

The second equality of (5.15) is equivalent to the first equality of (5.12).

We now rewrite (5.10) and (5.11) as

$$
\frac{\lambda_{i_{0}} \phi\left(n_{0}, i_{0}\right) P_{n_{0}}^{\phi^{\prime}}}{\mu_{n_{0}+1} P_{n_{0}+1}^{\phi}}=t_{1}-t_{2}
$$


and

$$
\frac{\lambda_{i_{0}}\left(1-\phi\left(n_{0}, i_{0}\right)\right) P_{n_{0}}^{\phi^{\prime \prime}}}{\mu_{n_{0}+1} P_{n_{0}+1}^{\phi}}=t_{4}-t_{3},
$$

respectively. Dividing (5.16) by (5.17) yields

$$
\frac{t_{1}}{t_{3}}=\frac{\left(t_{1}-t_{2}\right)\left(1-\phi\left(n_{0}, i_{0}\right)\right)}{\left(t_{4}-t_{3}\right) \phi\left(n_{0}, i_{0}\right)}=\frac{\left(t_{1}-1\right)\left(1-\phi\left(n_{0}, i_{0}\right)\right)}{\left(1-t_{3}\right) \phi\left(n_{0}, i_{0}\right)},
$$

where the second equality follows from (5.15). To conclude the proof, we observe that the equality

$$
\frac{t_{1}}{t_{3}}=\frac{\left(t_{1}-1\right)\left(1-\phi\left(n_{0}, i_{0}\right)\right)}{\left(1-t_{3}\right) \phi\left(n_{0}, i_{0}\right)}
$$

is equivalent to the second equality in (5.12).

Proof of Theorem 2.2. Since both $\phi^{\prime}$ and $\phi^{\prime \prime}$ are nonrandomized stationary policies and $\phi(n, i)=\phi^{\prime}(n, i)=\phi^{\prime \prime}(n, i)$ for any $(n, i) \neq\left(n_{0}, i_{0}\right)$, according to (2.14) either $x^{\phi}(n, i)=$ $x^{\phi^{\prime}}(n, i)=x^{\phi^{\prime \prime}}(n, i)=0$ or $x^{\phi}(n, i)=P_{n}^{\phi}, x^{\phi^{\prime}}(n, i)=P_{n}^{\phi^{\prime}}$, and $x^{\phi^{\prime \prime}}(n, i)=P_{n}^{\phi^{\prime \prime}}$ for any $(n, i) \neq\left(n_{0}, i_{0}\right)$. Thus, in view of Lemma 5.1 , to prove that $\left(x^{\phi}, P^{\phi}\right)$ is a convex combination of pairs $\left(x^{\phi^{\prime}}, P^{\phi^{\prime}}\right)$ and $\left(x^{\phi^{\prime \prime}}, P^{\phi^{\prime \prime}}\right)$, it suffices to show that the following three equalities hold:

$$
\begin{gathered}
\alpha x^{\phi^{\prime}}\left(n_{0}, i_{0}\right)+(1-\alpha) x^{\phi^{\prime \prime}}\left(n_{0}, i_{0}\right)=x^{\phi}\left(n_{0}, i_{0}\right), \\
\alpha P_{n_{0}}^{\phi^{\prime}}+(1-\alpha) P_{n_{0}}^{\phi^{\prime \prime}}=P_{n_{0}}^{\phi}, \\
\alpha P_{N}^{\phi^{\prime}}+(1-\alpha) P_{N}^{\phi^{\prime \prime}}=P_{N}^{\phi} .
\end{gathered}
$$

Since $\phi^{\prime}\left(n_{0}, i_{0}\right)=0$ and $\phi^{\prime \prime}\left(n_{0}, i_{0}\right)=1$, we have $x^{\phi^{\prime}}\left(n_{0}, i_{0}\right)=0$ and $x^{\phi^{\prime \prime}}\left(n_{0}, i_{0}\right)=P_{n_{0}}^{\phi^{\prime \prime}}$. We rewrite (5.18)-(5.20) as

$$
\begin{gathered}
(1-\alpha) P_{n_{0}}^{\phi^{\prime \prime}}=\phi\left(n_{0}, i_{0}\right) P_{n_{0}}^{\phi}, \\
(1-\alpha) \frac{P_{n_{0}}^{\phi^{\prime \prime}}}{P_{n_{0}}^{\phi}}+\alpha \frac{P_{n_{0}}^{\phi^{\prime}}}{P_{n_{0}}^{\phi}}=1, \\
(1-\alpha) \frac{P_{N}^{\phi^{\prime \prime}}}{P_{N}^{\phi}}+\alpha \frac{P_{N}^{\phi^{\prime}}}{P_{N}^{\phi}}=1 .
\end{gathered}
$$

These equalities can be further rewritten as

$$
\begin{gathered}
(1-\alpha) t_{3}=\phi\left(n_{0}, i_{0}\right), \\
(1-\alpha) t_{3}+\alpha t_{1}=1 \\
(1-\alpha) t_{4}+\alpha t_{2}=1
\end{gathered}
$$

Equalities (5.21), (5.22), and (5.23) respectively imply that

$$
\alpha=1-\frac{\phi\left(n_{0}, i_{0}\right)}{t_{3}}, \quad \alpha=\frac{1-t_{3}}{t_{1}-t_{3}}, \quad \text { and } \quad \alpha=\frac{t_{4}-1}{t_{4}-t_{2}} .
$$

In view of Lemma 5.2, all these values of $\alpha$ are equal. In addition, $0<\alpha<1$ because $t_{1}>1>t_{3}$. 


\section{Constrained optimization}

The goal of this section is to prove Theorem 2.3.

Proof of Theorem 2.3. Consider any 1-randomized stationary optimal policy $\phi$ for the problem (2.1). Due to Theorem 2.1(iii), the vector $\left(x^{\phi}, P^{\phi}\right)$ defined by (2.12)-(2.14) is an optimal solution to the LP (2.6). Let us consider the following two cases: (i) there exists a state $\left(n_{0}, i_{0}\right)$ for which $0<\phi\left(n_{0}, i_{0}\right)<1$; (ii) $\phi$ is nonrandomized. In case (i), Theorem 2.2 implies that there exist two stationary policies, $\phi^{\prime}$ and $\phi^{\prime \prime}$, that coincide with $\phi$ for all states, except for $\left(n_{0}, i_{0}\right)$, such that $\left(x^{\phi}, P^{\phi}\right)$ is a convex combination of the feasible solutions $\left(x^{\phi^{\prime}}, P^{\phi^{\prime}}\right)$ and $\left(x^{\phi^{\prime \prime}}, P^{\phi^{\prime \prime}}\right)$ to the LP (2.7). In addition, $\phi^{\prime}\left(n_{0}, i_{0}\right)=0$ and $\phi^{\prime \prime}\left(n_{0}, i_{0}\right)=1$. Since the LP (2.7) and the LP (2.11) have the same feasible regions, $\left(x^{\phi^{\prime}}, P^{\phi^{\prime}}\right)$ and $\left(x^{\phi^{\prime \prime}}, P^{\phi^{\prime \prime}}\right)$ are feasible for the LP (2.11). In view of Lemma 2.1, $\left(x^{\phi}, P^{\phi}\right)$ is optimal for the LP (2.11). Therefore, $\left(x^{\phi^{\prime}}, P^{\phi^{\prime}}\right)$ and $\left(x^{\phi^{\prime \prime}}, P^{\phi^{\prime \prime}}\right)$ are both optimal for the LP (2.11).

Consider the reward $r_{i}^{\prime}=r_{i}+\bar{u}_{1} c_{i}, i=1, \ldots, m$, defined prior to the introduction of the LP (2.11). According to Corollary 2.1, $\phi^{\prime}$ and $\phi^{\prime \prime}$ are both optimal stationary policies for the unconstrained problem (2.10), with rewards $r_{i}^{\prime}$. In view of Corollary 3.1, $\phi^{\prime}$ and $\phi^{\prime \prime}$ are trunk reservation policies consistent with the rewards $r_{i}^{\prime}$. Consider any type, $i$, such that $r_{i}^{\prime}<r_{i_{0}}^{\prime}$. Then $M_{i}^{\phi^{\prime}} \leq M_{i_{0}}^{\phi^{\prime}}$. Notice that both $\phi\left(n_{0}, i_{0}\right)>\phi^{\prime}\left(n_{0}, i_{0}\right)$ and $\phi(n, i)=\phi^{\prime}(n, i)$ when $(n, i) \neq$ $\left(n_{0}, i_{0}\right)$. Therefore, $M_{i}^{\phi} \leq M_{i_{0}}^{\phi}$. Similarly, for any type, $j$, such that $r_{j}^{\prime}>r_{i_{0}}^{\prime}$, we have $M_{j}^{\phi^{\prime \prime}} \geq M_{i_{0}}^{\phi^{\prime \prime}}$. Notice that both $\phi^{\prime \prime}\left(n_{0}, i_{0}\right)>\phi\left(n_{0}, i_{0}\right)$ and $\phi^{\prime \prime}(n, i)=\phi(n, i)$ when $(n, i) \neq$ $\left(n_{0}, i_{0}\right)$. Therefore, $M_{j}^{\phi} \geq M_{i_{0}}^{\phi}$ and $\phi$ is a randomized trunk reservation policy consistent with the rewards $r_{i}^{\prime}$. In case (ii), vector $\left(x^{\phi}, P^{\phi}\right)$ is optimal for the LP (2.11) and, by Corollary 3.1, $\phi$ is a trunk reservation policy consistent with $r^{\prime}$.

Example 6.1. Consider the $M / M / 1 /$ loss queue with three types of customer, as described in Example 3.2. In addition, set the costs to be $c_{1}=3, c_{2}=8$, and $c_{3}=1$ and the cost constraint to be $G=4.5$. By Theorem 2.1, any optimal policy for the constrained problem can be found by solving the LP (2.6), which here takes the following form:

maximize $2 x(0,1)+0.4 x(0,2)+0.6 x(0,3)$ over $x$ and $P$, subject to

$$
\begin{gathered}
3(1-x(0,1))+3.2(1-x(0,2))+0.6(1-x(0,3)) \leq 4.5, \\
x(0,1)+0.4 x(0,2)+0.6 x(0,3)=P_{1}, \\
P_{0}+P_{1}=1, \\
0 \leq x(0, i) \leq P_{0}, \quad i=1,2,3 .
\end{gathered}
$$

We shall show that any randomized trunk reservation policy $\phi_{k}$ defined by the thresholds $(1, k, 0), \frac{40}{57} \leq k \leq 1$, is optimal. Indeed, according to Example 3.2, the value of the above LP is not greater than 1. In addition, the last constraint and (2.8) together imply that $x(0,1)=P_{0}$, $x(0,2)=k P_{0}$, and $x(0,3)=0$ for any feasible policy $\phi_{k}, 0 \leq k \leq 1$. Therefore, the above LP implies that the best policy among the $\phi_{k}$ can be found by solving the following mathematical program:

$$
\begin{gathered}
\text { maximize } 2 P_{0}+0.4 k P_{0} \text { over } P_{0} \text { and } k, \text { subject to } \\
\qquad \begin{array}{c}
3 P_{0}+3.2 k P_{0} \geq 2.3, \\
2 P_{0}+0.4 k P_{0}=1, \\
P_{0} \geq 0 \\
0 \leq k \leq 1 .
\end{array}
\end{gathered}
$$


The constraint (6.3) and objective function (6.1) imply that the optimal value is indeed 1. Constraints (6.2)-(6.5) imply that any $k \in\left[\frac{40}{57}, 1\right]$ corresponds to the optimal solution $(x, P)$ to the original LP with $P_{0}=(2+0.4 k)^{-1}, P_{1}=1-P_{0}, x(0,1)=P_{0}, x(0,2)=k P_{0}$, and $x(0,3)=0$. Thus, we have proved that any policy $\phi_{k}, \frac{40}{57} \leq k \leq 1$, is optimal.

Referred to as 'shadow prices', Lagrange multipliers measure the rate of change of the optimum objective function value with respect to changes in the constraints [10, p. 37]. In this example, since any small perturbation of the constraint $G$ in (2.2) does not change the optimum objective function value, $\bar{u}_{1}$ equals 0 and is unique. Theorem 2.3 implies that $r_{1}^{\prime}=2>r_{2}^{\prime}=r_{3}^{\prime}=1$. It is easy to verify that any randomized trunk reservation policy $\psi$ with $\left(M_{1}^{\psi}, M_{2}^{\psi}, M_{3}^{\psi}\right)=(1, s, 1), 0 \leq s \leq 1$, is unfeasible. Therefore, although $r_{1}^{\prime}>r_{3}^{\prime}=r_{2}^{\prime}$, there exists no optimal randomized trunk reservation policy such that $M_{1} \geq M_{3} \geq M_{2}$. This implies that there exists no stationary optimal policy $\phi$ defined by thresholds satisfying $M_{i}^{\phi} \geq M_{j}^{\phi}$ when $r_{i}^{\prime} \geq r_{j}^{\prime}$. We recall that, according to Theorem 2.3, for a feasible problem there exists a stationary optimal policy $\phi$ defined by thresholds satisfying the inequalities $M_{i}^{\phi} \geq M_{j}^{\phi}$ when $r_{i}^{\prime}>r_{j}^{\prime}$.

\section{Acknowledgements}

This research was partially supported by NSF grant DMI-0300121. The authors thank E. Alper Yildirim for his insightful comments on Appendix B in the preliminary version of this paper, which led to a more general formulation of Lemma B.1.

\section{Appendix A. Proof of Lemma 3.3}

We first recall the notation used in the key theorem of [9]. A corollary of it is needed in the proof of Lemma 3.3.

From [9], the definitions of the sets of policies $\Delta^{1}, \Delta^{2}$, and $\Delta^{3}$ are as follows. By $\Delta^{1}$ we denote the set of randomized stationary policies that satisfy the inequalities

$$
\phi(n, j) \geq \phi(n, j+1), \quad n=0, \ldots, N-1, j=1, \ldots, m-1,
$$

and the condition that, for each $n=0, \ldots, N-1$, all but at most one of the probabilities $\phi(n, j), j=1, \ldots, m$, equal 0 or 1 . By $\Delta^{2}$ we denote the set of randomized stationary policies that satisfy

$$
\phi(n, 1)=1, \quad n=0, \ldots, N-1 .
$$

By $\Delta^{3}$ we denote the set of randomized stationary policies that satisfy the condition

$$
\phi(n, j) \geq \phi(n+1, j), \quad n=0, \ldots, N-2, j=1, \ldots, m,
$$

and the condition that, for each $j=1, \ldots, m$, all but at most one of the probabilities $\phi(n, j)$, $n=0, \ldots, N-1$, equal 0 or 1 .

Lemma A.1. (Theorem 3.2 of [9].) Given $r_{1}, r_{2}, \ldots, r_{m}, r_{1}>r_{2}>\cdots>r_{m}$, let $\phi$ be an optimal randomized stationary policy (for problem (1.7)-(1.8) of [9]). Then $\phi \in \Delta^{1} \cap \Delta^{2} \cap \Delta^{3}$.

By letting the right-hand side of the constraint (1.8) of [9] equal 1 in Lemma A.1, we obtain the following result for an unconstrained problem.

Corollary A.1. Let $\phi$ be a randomized stationary optimal policy for an unconstrained problem with $r_{1}>r_{2}>\cdots>r_{m}$. Then $\phi \in \Delta^{1} \cap \Delta^{2} \cap \Delta^{3}$. 
Proof of Lemma 3.3. (i) If the rewards are all distinct, this lemma holds due to Corollary A.1. Otherwise, consider the situation in which there are $K$ different rewards satisfying $r_{1}^{*}>r_{2}^{*}>\cdots>r_{K}^{*}, K \in\{1, \ldots, m-1\}$. Let $I_{k}$ be the set of customer types whose rewards are equal to $r_{k}^{*}, k=1, \ldots, K$, and define $\Lambda_{k}=\sum_{i \in I_{k}} \lambda_{i}$. Due to Feinberg [8, Section 4], all customer types with equal rewards can be merged together without loss of optimality. Define a randomized stationary policy by

$$
\psi(n, k):=\sum_{i \in I_{k}} \phi(n, i) \frac{\lambda_{i}}{\Lambda_{k}}, \quad k=1, \ldots, K, n=1, \ldots, N-1,
$$

for the smaller-sized problem with combined classes. According to (2.12) and (2.13), $P_{n}^{\phi}=P_{n}^{\psi}$ for all $n$. Therefore, $W(\phi)=W(\psi)$ and $\psi$ is an optimal randomized stationary policy for the problem (2.10). Therefore, $\psi$ is also optimal for the problem with $K$ customer classes with distinct rewards. In view of Corollary A.1, we have $\psi \in \Delta^{1} \cap \Delta^{2} \cap \Delta^{3}$ in the problem with $K$ customer types. Since $\psi \in \Delta^{1}$ in the model with $K$ customer classes, (i) and (ii) hold. Similarly, having $\psi \in \Delta^{2}$ implies (iii) and having $\psi \in \Delta^{3}$ implies (iv).

\section{Appendix B. Lagrangian relaxation}

In this appendix we present the results on Lagrangian optimization in convex and linear programming used in this paper. Let us consider a mathematical programming problem $P$ :

$$
\begin{aligned}
\operatorname{minimize} f(x) & \text { subject to } \\
g_{i}(x) & \leq 0, \quad i=1, \ldots, s, \\
h_{i}(x) & =0, \quad i=1, \ldots, p, \\
x & \in \mathbb{R}^{n} .
\end{aligned}
$$

The problem $P$ is a convex programming problem if (i) $f$ and $g_{1}, \ldots, g_{s}$ are convex functions and (ii) $h_{1}, \ldots, h_{p}$ are linear functions. If the functions $f, g_{i}, i=1, \ldots, s$, and $h_{i}, i=$ $1, \ldots, p$, are all linear, then problem $P$ becomes a linear programming problem.

The set

$$
S=\left\{x \in \mathbb{R}^{n}: g_{i}(x) \leq 0, i=1, \ldots, s ; h_{i}(x)=0, i=1, \ldots, p\right\}
$$

is called the feasible set and any $x \in S$ is called a feasible point. We consider the vectorvalued functions $g=\left(g_{1}, \ldots, g_{s}\right)^{\top}$ and $h=\left(h_{1}, \ldots, h_{p}\right)^{\top}$. For a vector-valued function $F(x)=\left(F_{1}(x), \ldots, F_{N}(x)\right)^{\top}$ defined for some $x \in \mathbb{R}^{n}$, where $N=1,2, \ldots$, we denote by $\nabla F(x)$ the $n \times N$ gradient matrix with the elements $\partial F_{i}(x) / \partial x_{i}$, whenever all these partial derivatives exist at the point $x=\left(x_{1}, \ldots, x_{n}\right)$. The following two statements are well known [20, p. 201]: (i) if $\nabla F(x)$ exists at $x$ and $\nabla F$ is continuous at $x$, then $F$ is differentiable at $x$, and (ii) if $F$ is differentiable at $x$ then it is continuous at $x$ and $\nabla F(x)$ exists.

For two vectors, $a$ and $b$, of equal dimensions, we denote their scalar product by $a b$. We also write $a \geq b, a=b$, and $a \leq b$ if the corresponding relations hold for all the corresponding coordinates.

Define two row vectors, $u=\left(u_{1}, \ldots, u_{s}\right)$ and $v=\left(v_{1}, \ldots, v_{p}\right)$. The function

$$
L(x, u, v)=f(x)+u g(x)+v h(x)
$$

is called the Lagrangian function. 
Definition B.1. (Karush-Kuhn-Tucker (KKT) point [20, p. 94].) A point $(\bar{x}, \bar{u}, \bar{v})$, where $\bar{x} \in \mathbb{R}^{n}, \bar{u} \in \mathbb{R}^{s}$, and $\bar{v} \in \mathbb{R}^{p}$, is called a KKT point for the mathematical programming problem $P$ if the vector functions $f, g$, and $h$ are differentiable at $\bar{x}=\left(\bar{x}_{1}, \ldots, \bar{x}_{n}\right)$ and

$$
\begin{aligned}
\nabla_{x} L(\bar{x}, \bar{u}, \bar{v}) & :=\nabla f(\bar{x})+\bar{u} \nabla g(\bar{x})+\bar{v} \nabla h(\bar{x})=0, \\
g(\bar{x}) & \leq 0, \\
h(\bar{x}) & =0, \\
\bar{u} & \geq 0, \\
\bar{u} g(\bar{x}) & =0 .
\end{aligned}
$$

The vectors $\bar{u}=\left(\bar{u}_{1}, \ldots, \bar{u}_{s}\right)$ and $\bar{v}=\left(\bar{v}_{1}, \ldots, \bar{v}_{p}\right)$ are called Lagrange multipliers. Sometimes we say that $\bar{u}_{i}$ and $\bar{v}_{i}$ are the Lagrange multipliers with respect to the constraints $g_{i}(x) \leq 0$ and $h_{i}(x)=0$, respectively. Note that the values of $\bar{u}_{i}$ and $\bar{v}_{i}$ might not be unique.

Constraint qualification [20, p. 171] for the functions $g$ and $h$ plays an important role in nonlinear programming. However, since we deal only with linear programming in this paper, constraint qualification holds at any minimal point (local minima are global minima in linear programming). Therefore, we will not introduce the definition of constraint qualification here.

Condition B.1. (First-order necessary optimality condition [20, p. 173].) Suppose that (i) $\bar{x}$ is a local minimal solution to problem $P$, (ii) the vector functions $f, g$, and h are differentiable at $\bar{x}$, and (iii) constraint qualification holds at $\bar{x}$ for $g$ and $h$. Then there exist points $\bar{u}$ and $\bar{v}$, where $\bar{u} \in \mathbb{R}^{s}$ and $\bar{v} \in \mathbb{R}^{p}$, such that $(\bar{x}, \bar{u}, \bar{v})$ is a KKT point.

Condition B.2. (First-order sufficient optimality condition [20, p. 162].) Suppose that $P$ is a convex programming problem. If a KKT point $(\bar{x}, \bar{u}, \bar{v})$ exists then $\bar{x}$ is a global minimum.

If the objective is to maximize $f(x)$, we observe that

$$
\max f(x)=-\min \{-f(x)\} .
$$

The corresponding Lagrangian function is

$$
L(x, u, v)=-\left(-f(x)+u^{\top} g(x)+v^{\top} h(x)\right)=f(x)-u^{\top} g(x)-v^{\top} h(x) .
$$

Since, in this paper, the functions $f, g$, and $h$ are linear, the corresponding negative functions are also linear and, therefore, convex.

Lemma B.1. Let $(\bar{x}, \bar{u}, \bar{v})$ be a KKT point of a convex programming problem $P$. Consider problem $B$ :

$$
\begin{aligned}
\text { minimize } & f(x)+\bar{u}^{k} g(x)^{k} \text { subject to } \\
g_{i}(x) & \leq 0, \quad i=k+1, \ldots, s, \\
h_{i}(x) & =0, \quad i=1, \ldots, p, \\
x & \in \mathbb{R}^{n},
\end{aligned}
$$

where $g^{k}=\left(g_{1}, \ldots, g_{k}\right)^{\top}, \bar{u}^{k}=\left(\bar{u}_{1}, \ldots, \bar{u}_{k}\right)$, and $k<s$. Problems $P$ and $B$ then have the same optimal values, and any optimal solution to problem $P$ is an optimal solution to problem $B$.

Proof. Consider a KKT point $(\bar{x}, \bar{u}, \bar{v})$ of problem $P$. Equations (B.1)-(B.5) imply that $\left(\bar{x}, u_{k+1}, \ldots, u_{s}, \bar{v}\right)$ is a KKT point for problem $B$. According to the first-order sufficient optimality condition, $\bar{x}$ is a global minimizer for both problems $P$ and $B$. Equation (B.5) shows that problems $P$ and $B$ have the same optimal value. 
When problem $P$ is an LP, $(\bar{x}, \bar{u}, \bar{v})$ is a KKT point if and only if $\bar{x}$ is an optimal solution to $P$ and $(\bar{u}, \bar{v})$ is an optimal solution to the problem dual to $P[20, \mathrm{pp} .115,127]$. Thus, to find $\bar{u}^{k}$ we need to solve the LP dual to $P$. However, most contemporary LP solvers use interior-point methods and calculate the primary and dual solutions simultaneously. Therefore, we do not formulate the dual LP here.

\section{References}

[1] Altman, E. (2002). Applications of Markov decision processes in telecommunication networks. In Handbook of Markov Decision Processes, eds E. Feinberg and A. Schwartz, Kluwer, Boston, MA, pp. 480-536.

[2] Altman, E., Jimenez, T. And Koole, G. (2001). On optimal call admission control in a resource-sharing system. IEEE Trans. Commun. 49, 1659-1668.

[3] Carrizosa, E., Conde, E. and Munoz-Márquez, M. (1998). Admission policies in loss queueing models with heterogeneous arrivals. Manag. Sci. 44, 311-320.

[4] Crabill, T. B., Gross, D. and Magazine, M. J. (1977). A classified bibliography of research on optimal design and control of queues. Operat. Res. 25, 219-232.

[5] Feinberg, E. A. (1994). Constrained semi-Markov decision processes with average rewards. Z. Operat. Res. 39, 257-288.

[6] Feinberg, E. A. (2002). Constrained finite continuous-time Markov decision processes with average rewards. In Proc. IEEE 2002 Conf. Decisions Control (December 2002, Las Vegas), pp. 3805-3810.

[7] Feinberg, E. A. (2004). Continuous time discounted jump Markov decision processes: a discrete-event approach. Math. Operat. Res. 29, 492-524.

[8] Feinberg, E. A. (2005). On essential information in sequential decision processes. Math. Meth. Operat. Res. 62, 399-410.

[9] Feinberg, E. A. and Reiman, M. I. (1994). Optimality of randomized trunk reservation. Prob. Eng. Inf. Sci. 8, 463-489.

[10] Fiacco, A. V. AND McCoRmick, G. P. (1990). Nonlinear Programming: Sequential Unconstrained Minimization Techniques. SIAM, Philadelphia, PA.

[11] Hunt, P. J. AND Laws, C. N. (1997). Optimization via trunk reservation in single resource loss systems under heavy traffic. Ann. Appl. Prob. 7, 1058-1079.

[12] Kelly, F. P. (1990). Routing and capacity allocation in networks with trunk reservation. Math. Operat. Res. 15, 771-793.

[13] Key, P. (1990). Optimal control and trunk reservation in loss networks. Prob. Eng. Inf. Sci. 4, $203-242$.

[14] Lewis, M. E. (2001). Average optimal policies in a controlled queueing system with dual admission control. J. Appl. Prob. 38, 369-385.

[15] Lewis, M. E., Ayhan, H. And Foley, R. D. (1999). Bias optimality in a queue with admission control. Prob. Eng. Inf. Sci. 13, 309-327.

[16] Lewis, M. E., Ayhan, H. And Foley, R. D. (2002). Bias optimal admission policies for a nonstationary multiclass queueing system. J. Appl. Prob. 39, 20-37.

[17] Lin, K. Y. AND Ross, S. M. (2003). Admission control with incomplete information of a queueing system. Operat. Res. 51, 645-654.

[18] Lin, K. Y. And Ross, S. M. (2004). Optimal admission control for a single-server loss queue. J. Appl. Prob. 41, 535-546.

[19] Lippman, S. A. (1975). Applying a new device in the optimization of exponential queuing systems. Operat. Res. 23, 687-710.

[20] Mangasarian, O. L. (1969). Nonlinear Programming. McGraw-Hill, New York.

[21] Miller, B. L. (1969). A queueing reward system with several customer classes. Manag. Sci. 16, $235-245$.

[22] Nguyen, V. (1991). Optimality of trunk reservation in overflow process. Prob. Eng. Inf. Sci. 5, 369-390.

[23] Papastavrou, J. D., Rajagopalan, S. and Kleywegt A. J. (1996). The dynamic and stochastic knapsack problem with deadlines. Manag. Sci. 42, 1706-1718.

[24] PiunovskiY, A. B. (2004). Bicriteria optimization of a queue with a controlled input stream. Queueing Systems 48, 159-184.

[25] Puhalskit, A. A. And Reiman, M. I. (1998). A critically loaded multirate link with trunk reservation. Queueing Systems 28, 157-190.

[26] Ross, K. W. (1995). Multiservice Loss Models for Broadband Telecommunication Networks. Springer, London.

[27] Ross, K. W. AND YaO, D. D. (1990). Monotonicity properties for the stochastic knapsack. IEEE Trans. Inf. Theory 36, 1173-1179.

[28] Stidham, S., Jr. (1985). On optimal control of admission to a queueing system. IEEE Trans. Automatic Control 30, 705-713. 